\title{
Creación de un banco genómico de esperma para la conservación de la rana coronada (Triprion spinosus)
}

\author{
Rodríguez, Karina \\ Instituto Smithsonian de Investigaciones Tropicales \\ Panamá, Panamá \\ k_r_sanchez@hotmail.com \\ Arcia, Igli \\ Instituto Smithsonian de Investigaciones Tropicales \\ Panamá, Panamá \\ arciaigli@hotmail.com \\ Della Togna, Gina \\ Instituto Smithsonian de Investigaciones Tropicales \\ Panamá, Panamá \\ gina.dellatogna@uip.pa
}

\begin{abstract}
A worldwide rapid decrease in amphibian species diversity during the past decades requires urgent conservation strategies. The spiny-headed tree frog (Anotheca spinosa) belongs to this family. The main threats for the species include habitat loss and degradation and Bd. Genome Resource Banks (GRB's) serve as insurance policies for threatened species GRB's can be applied to store germplasm of threatened species or populations, with the intention of using it for reproduction purposes in the future. The objective of this study is to develop a hormonal stimulation and sperm cryopreservation protocol for the conservation of this species. Hormonal stimulation was first applied on 35 males. Treatments were applied via intraperitoneal injection. Eight (8) samples of spermic urine were collected for each individual gently introducing a catheter in the cloacae. To establish the sperm production peak for each hormone, samples were collected at 0.5, 1.5, 2.5, 3.5, 4.5, 5.5, 6.5, 24,28 y $48 \mathrm{~h}$ post-injection. Hormonal treatments consisted in 1, 2 and $4 \mu \mathrm{g} / \mathrm{gbw}$ of GnRH-desGly10, D-Ala6, Pro-NHEt9; and 5 y $10 \mathrm{lU} / \mathrm{gbw}$ of hCG. Urine without any stimulation or after injection with Amphibian Ringer Solution (ARS) were used as controls. Sperm quality in terms of concentration, osmolality, $\mathrm{pH}$, motility, volume and forward progressive motility was analyzed at each sperm collection. Results indicate that all used treatments stimulate sperm production in A. spinosa, nevertheless, treatments with 1 and $4 \mu \mathrm{g} / \mathrm{gpc} \mathrm{GnRH}$ were
\end{abstract}


not different to controls.

Keywords: amphibian, conservation, genomic bank, criopreservation, hormonal stimulation

\section{Resumen}

Una rápida disminución de la diversidad de especies de anfibios que se ha producido en todo el mundo en las últimas décadas requiere medidas de conservación inmediata y eficientes. La rana arbórea coronada (Anotheca spinosa). La especie está siendo afectada por el hongo causante de la quitridiomicosis ( $\mathrm{Bd}$ ). Los bancos genómicos (GRB) funcionan como pólizas de seguro para las especies en peligro Los GRB se pueden utilizar para almacenar germoplasma de poblaciones amenazadas, con la intención deliberada de utilizarlos en un programa de reproducción en el futuro. El objetivo de este estudio es desarrollar un protocolo de estimulación hormonal y criopreservación de esperma para la conservación de la Rana Coronada (Anotheca spinosa). Se aplicó la estimulación hormonal en 35 machos de A. spinosa. Los tratamientos fueron aplicados vía intraperitoneal. Se tomaron muestras de 8 individuos por tratamiento. La colecta de la orina espermática se llevó a cabo insertando un catéter en la cloaca. Para establecer las curvas de concentración espermática y los picos de producción para cada hormona, las muestras fueron colectadas 0.5, 1.5, 2.5, 3.5, 4.5, $5.5,6.5,24,28$ y $48 \mathrm{~h}$ post-estimulación. Los tratamientos hormonales utilizados fueron: 1, $2 \circ 4 \mu \mathrm{g} / \mathrm{gpc}$ del agonista de la hormona liberadora de la gonadotropina GnRH-desGly10, D-Ala6, Pro-NHEt9; 5 y 10 IU/gpc hormona gonadotrópica humana (hCG). Como controles se utilizó orina obtenida sin estimulación hormonal y controles de orina de animales inyectados con Amphibian Ringer Solution (ARS). Para cada una de las colectas se procedió a evaluar la calidad espermática en términos de concentración, osmolalidad, $\mathrm{pH}$, volumen, porcentaje de motilidad, porcentaje de movimiento progresivo y morfología). Los resultados indican que para la especie $A$. espinosa todos los tratamientos hormonales estimularon la producción de esperma, sin embargo, los tratamientos con 1 y $4 \mu \mathrm{g} / \mathrm{gpc}$ $\mathrm{GnRH}$ no fueron diferentes a los controles.

Palabras claves: anfibio, conservación, banco genómico, criopreservación, estimulación hormonal. 


\section{INTRODUCCIÓN}

La clase anfibia es el grupo de vertebrados que actualmente está enfrentando serias amenazas, llevando a muchas especies a la extinción, trayendo como consecuencia drásticas disminuciones que están siendo predominantes en los trópicos de Mesoamérica, América del Sur y Australia [1], [2]. La modificación del hábitat, contaminación y las enfermedades emergentes como Batrachochytrium dendrobatidis $(\mathrm{Bd})$ son las causas principales que amenazan con extinguir a muchas especies de anfibios [3]-[6]. En la actualidad para los anfibios se han identificado cerca de 8,360 especies; siendo el orden Anura, el grupo con mayor número de especies, 7,381, mientras que, en el orden Caudata, se encuentran alrededor de 766 especies y para el orden Gymnophiona, se han reportado un total de 213 especies [7]. Sin embargo, el $41 \%$ de las especies que se han descrito se encuentran bajo alguna amenaza que aumenta el riesgo de extinción [2], [8]. Todas estás amenazas requieren la aplicación de nuevas tecnologías, como lo son las técnicas de reproducción asistida (ART) que buscan mitigar los riesgos de futuras extinciones, aplicando técnicas como la criopreservación de recursos genéticos [9]

\section{MÉTODO}

\section{A. ESTIMULACIÓN HORMONAL}

Este proceso se llevó a cabo en el laboratorio del Instituto Smithsonian de Investigaciones Tropicales, ubicado en Gamboa. Se inyectaron 35 machos de Triprion spinosus, la misma fue aplicada de manera intraperitoneal a un ángulo de 450 en dirección craneal del animal. Se tomaron muestras de 8 individuos por tratamiento. La colecta de la orina espermática se llevó a cabo insertando un catéter en la cloaca (Micro Medical Tubing, 85 Durometer Vinyl). Para establecer las curvas de concentración espermática y los picos de producción para cada hormona, las muestras fueron colectadas $0.5,1.5,2.5,3.5,4.5,5.5,6.5,24,28$ y $48 \mathrm{~h}$ post-estimulación. Los tratamientos hormonales utilizados fueron:

- 1, 2 or $4 \mu \mathrm{g} / \mathrm{gpc}$ del agonista de la hormona liberadora de la gonadotropina GnRHdes-Gly10, D-Ala6, Pro-NHEt9

- 5 y 10 IU/gpc hormona gonadotrópica humana (hCG).

Como controles para evaluar la eficiencia de la estimulación hormonal y los parámetros espermáticos se utilizó orina obtenida sin estimulación hormonal, se utilizó un total de 4 controles, de estos 2 sin ninguna inyección y 2 controles inyectados con Amphibian Ringer Solution (ARS).

\section{B. ANÁLISIS DE MUESTRAS}

Las muestras fueron colocadas en tubos Eppendorf rotulados y se evaluaban de la siguiente manera: 
- Se observó por medio del microscopio y utilizando la Cámara de Neubauer (Hausser ${ }^{\mathrm{TM}}$ Bright-Line $^{\mathrm{TM}}$ Phase Hemacytometer) con un cubre objetos (Hausser Scientific ${ }^{\mathrm{TM}}$ Hemacytometer Cover Glass) y se analizó, la motilidad, movimiento progresivo y concentración de los espermatozoides (si la muestra se encontraba muy concentrada se diluía 1:10 en Solución Ringer de anfibios).

- El volumen total de orina espermática se calculó por medio de una pipeta de 10, 200 o 1000 microlitros.

- Utilizando un osmómetro se midió la osmolalidad y con tiras de pH se midió el valor numérico del pH de cada colecta en cada individuo.

Una vez obtenidos los datos de la estimulación hormonal, estos fueron analizados utilizando el estadístico de ANOVA para determinar las diferencias significativas entre tratamientos.

\section{RESULTADOS}

\section{A. Curvas de estimulación hormonal por tratamiento}

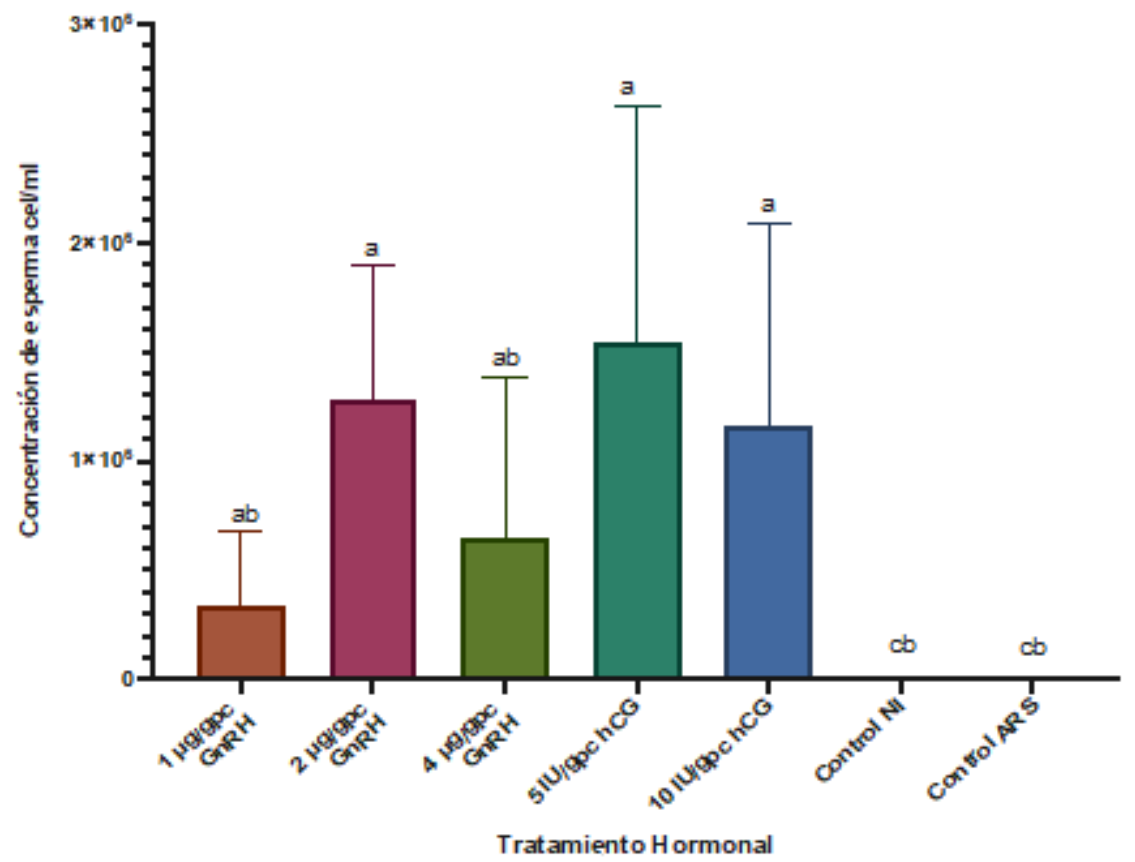

Fig 1. Análisis del efecto de los tratamientos hormonales en la producción de esperma en términos de concentración (células por $\mathrm{ml}$ ).

Los datos de concentración de esperma fueron análizados mediante el estadístico de Kruskal-Wallis. El gráfico indica que los tratamientos son efectivos estimulando la producción de esperma en comparación a los controles [Kruskal-Wallis H (7): 41.11; $p<0.0001$ ]. 


\section{B. Curvas hormonales}

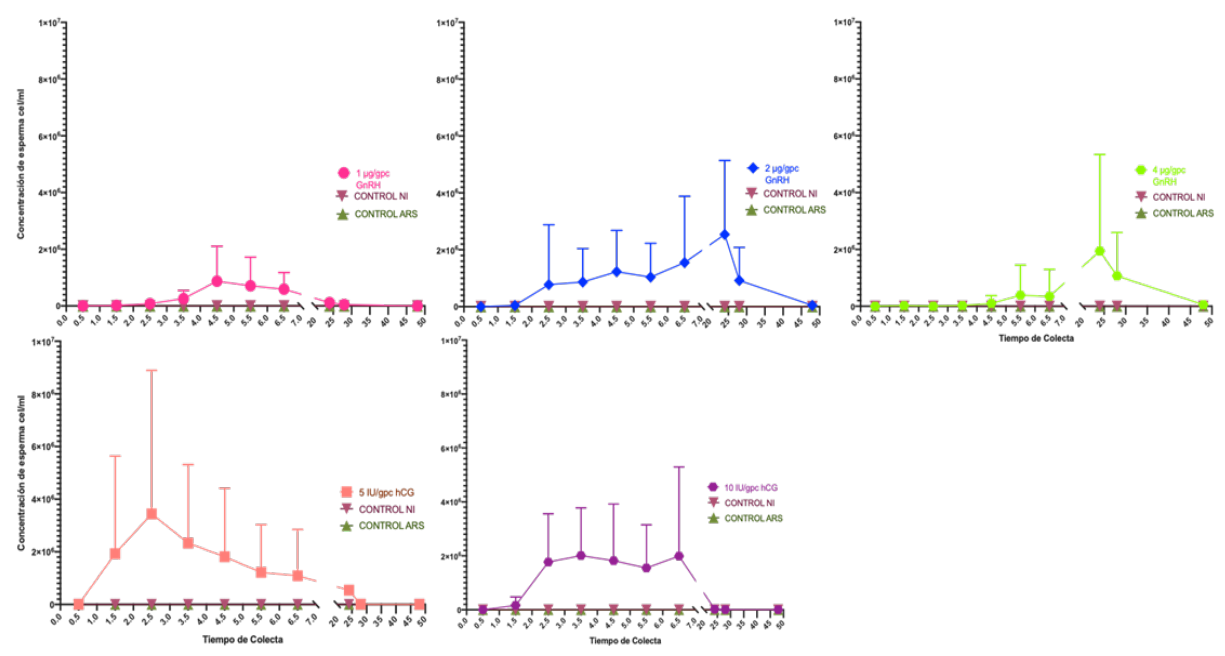

Fig 2. Curvas hormonales por tiempo de colecta

El gráfico 2 muestra los datos de concentración de esperma por punto de tiempo de colecta, resultando en las curvas de producción de esperma por tratamiento hormonal, mostrando los picos de producción. Las muestras fueron colectadas a 0.5, 1.5, 2.5, 3.5, 4.5, $5.5,6.5,24,28$ y 48 h post-estimulación. Para cada tiempo de colecta se promediaron las concentraciones y me muestran expresados como media $\pm \mathrm{DE}$.

\section{Caracterización de células espermáticas}

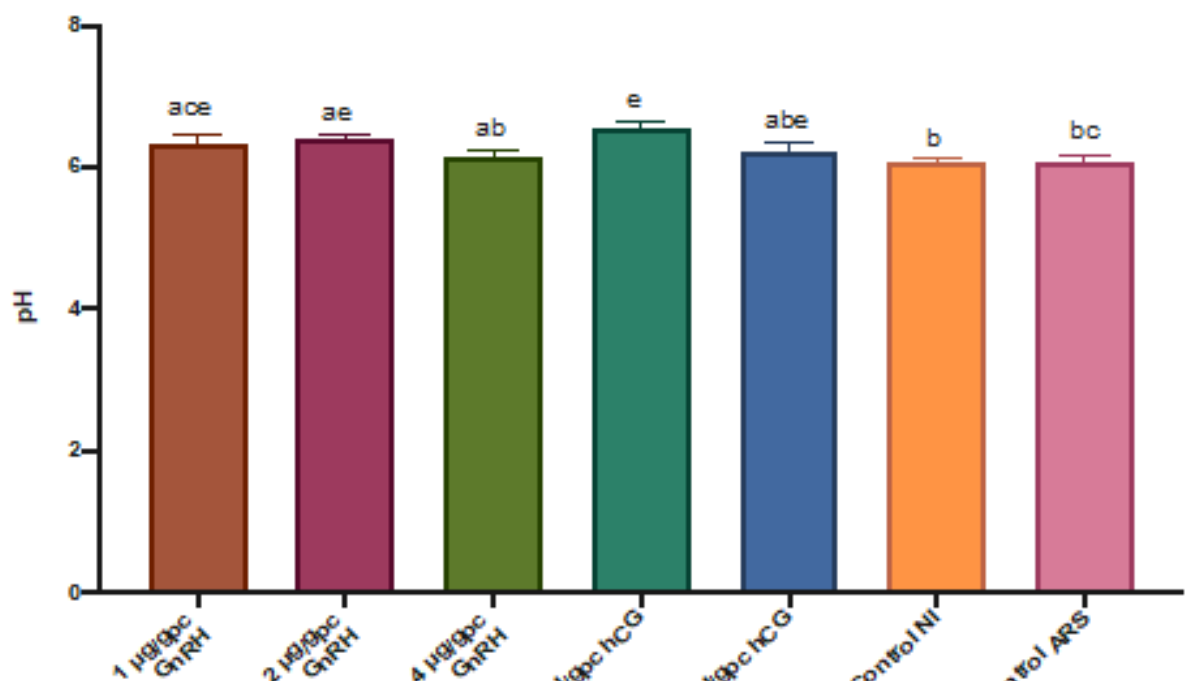

Fig 3. Análisis del efecto de los tratamientos hormonales en el pH de la orina espermática. 
El gráfico indica que algunos tratamientos difieren de los controles, elevando el pH de la orina espermática. Tratamiento con $1 \mathrm{mg} / \mathrm{gpc} \mathrm{GnRH}, 2 \mathrm{mg} / \mathrm{gpc} \mathrm{GnRH}$ y 5 Ul/gpc hCG muestran pH más elevado que el control sin inyección, mientras que tratamiento con 2 $\mathrm{mg} / \mathrm{gpc} \mathrm{GnRH}$ y $5 \mathrm{UI} / \mathrm{gpc}$ hCG muestran pH más elevado que el control ARS. Todos los tratamientos, a excepción de $5 \mathrm{UI} / \mathrm{gpc}$ hCG, no muestran diferencia en el pH de la orina espermática [Kruskal-Wallis H (7): 40.64; $\mathrm{p}<0.0001$ ].
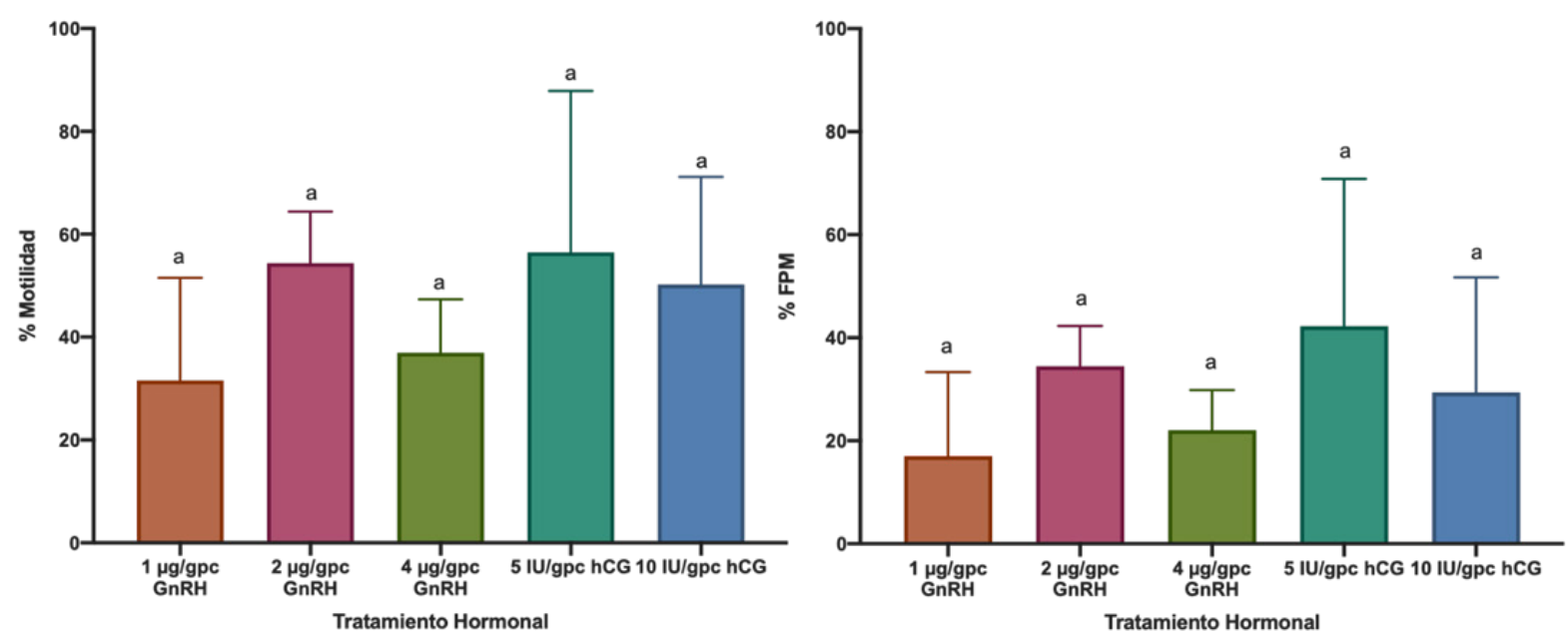

Fig 4. Análisis del efecto de los tratamientos hormonales en la motilidad y movimiento progresivo de las células espermáticas.

El gráfico indica que no existe diferencia significativa en el porcentaje de motilidad entre los tratamientos, observándose una tendencia de los tratamientos con $2 \mathrm{mg} / \mathrm{gpc} \mathrm{GnRH}, 5$ UI/gpc hCG y $10 \mathrm{UI} / \mathrm{gpc}$ hCG a estimular la producción de esperma con mayor porcentaje de motilidad [Kruskal-Wallis $\mathrm{H}(5)$ : 7.837; $p>0.05$ ]. De igual forma, no hubo diferencia entre tratamientos con respecto al porcentaje de células que muestran movimiento progresivo. Nuevamente, tratamiento con 2 mg/gpc GnRH, 5 UI/gpc hCG y $10 \mathrm{UI} / \mathrm{gpc}$ hCG muestran una tendencia a estimular la producción de células con mayor porcentaje de movimiento progresivo [Kruskal-Wallis H (5): 6.624; $p>0.05$ ]. 


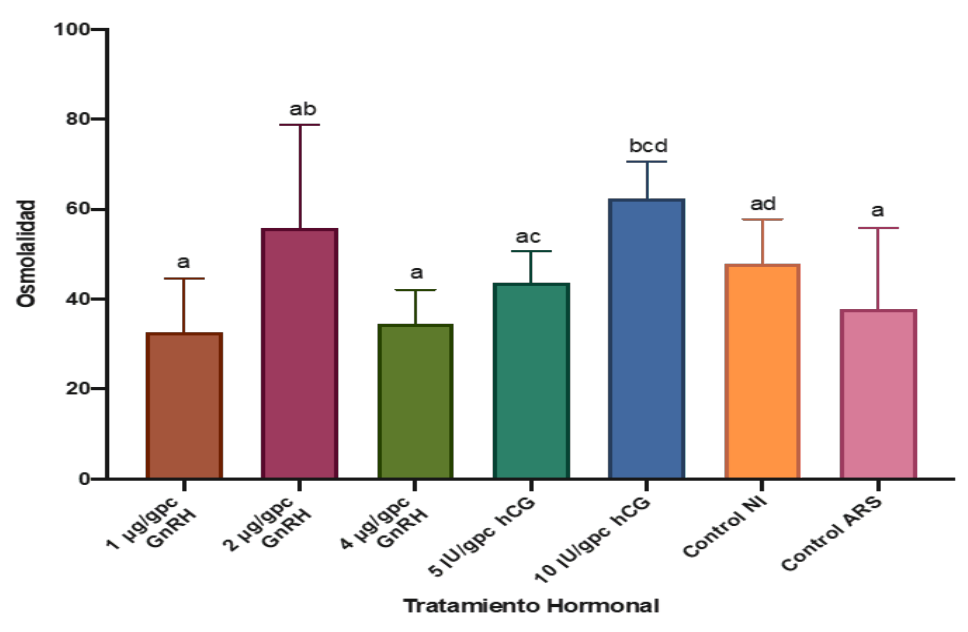

Fig 5. Análisis del efecto de los tratamientos hormonales en la osmolalidad de la orina espermática.

El gráfico indica que tratamiento con $10 \mathrm{UI} / g p c$ hCG aumenta la osmolalidad de la orina espermática en comparación con los tratamientos con $1 \mathrm{mg} / \mathrm{gpc} \mathrm{GnRH}, 4 \mathrm{mg} / \mathrm{gpc} \mathrm{GnRH}$ y el control con ARS. A excepción de $10 \mathrm{UI} / \mathrm{gpc}$ hCG, ningún tratamiento muestra diferencia con los controles.

\section{CONCLUSIONES}

A partir de los resultados de estimulación hormonal, concluimos que la especie $T$. spinosus responde positivamente a la estimulación con la hormona GnRH y hCG, entre estos, $2 \mu \mathrm{g} / \mathrm{gpc} \mathrm{GnRH}, 5 \mathrm{UI} / \mathrm{gpchCG}$ y $10 \mathrm{Ul} / \mathrm{gpc}$ hCG proporcionan esperma de mejor calidad. Por medio de estos resultados se logró identificar el tiempo de acción y los picos de cada uno de los tratamientos hormonales efectivos. Proporcionamos información, hasta ahora inexistente, sobre el efecto de la estimulación hormonal en la producción de esperma de los individuos en cautiverio de la especie Triprion spinosus.

\section{Referencias}

[1] A. B. C. Scheele, F. Pasmans, L. F. Skerratt, L. Berger, and A. Martel, "Title : Amphibian fungal panzootic causes catastrophic and ongoing loss of biodiversity," pp. 1-59.

[2] G. Ceballos, P. R. Ehrlich, and P. H. Raven, "Vertebrates on the brink as indicators of biological annihilation and the sixth mass extinction," Proc. Natl. Acad. Sci. U. S. A., vol. 117, no. 24, pp. 13596-13602, 2020, doi: 10.1073/pnas.1922686117.

[3] K. R. Lips, J. D. Reeve, and L. R. Witters, "Ecological Traits Predicting Amphibian Population Declines in Central America," Conserv. Biol., vol. 17, no. 4, pp. 1078-1088, 2003, doi: 10.1046/j.15231739.2003.01623.x.

[4] D. M. Green, "Biology of amphibian declines," Amphib. Declines Conserv. Status United States Species, no. May, pp. 28-33, 2005, doi: 10.1525/california/9780520235922.003.0007. 
[5] G. B. R. and J. G. M. P. J. Bishop, A. Angulo, J. P. Lewis, R.D. Moore, "The Amphibian Extinction Crisis - what will it take to put the action into the Amphibian Conservation Action Plan?," S.A.P.I.EN.S, vol. 5, no. August, 2012.

[6] L. Berger et al., "Chytridiomycosis causes amphibian mortality associated with population declines in the rain forests of Australia and Central America," Proc. Natl. Acad. Sci. U. S. A., vol. 95, no. 15, pp. 9031-9036, 1998, doi: 10.1073/pnas.95.15.9031.

[7] D. R. Frost, “Amphibian Species of the World: an Online Reference," version 6.1, 2021. https:// amphibiansoftheworld.amnh.org/index.php. (accessed May 20, 2021).

[8] IUCN, "The IUCN Red List of Threatened Species," Versión 2021-1, 2021. .

[9] A. J. Kouba et al., "Emerging trends for biobanking amphibian genetic resources: The hope, reality and challenges for the next decade," Biol. Conserv., vol. 164, pp. 10-21, 2013, doi: 10.1016/j. biocon.2013.03.010.

\section{Autorización y Licencia CC}

Los autores autorizan a APANAC XVIII a publicar el artículo en las actas de la conferencia en Acceso Abierto (Open Access) en diversos formatos digitales (PDF, HTML, EPUB) e integrarlos en diversas plataformas online como repositorios y bases de datos bajo la licencia CC:

Attribution-NonCommercial-ShareAlike 4.0 International (CC BY-NC-SA 4.0) https://creativecommons. org/licenses/by-nc-sa/4.0/.

Ni APANAC XVIII ni los editores son responsables ni del contenido ni de las implicaciones de lo expresado en el artículo. 